\title{
Adventitial Cystic Disease of the Superficial Femoral Vein without a Joint Connection: A Case Report
}

\author{
Young Sup Yoo ${ }^{1}$, Yoo Duk Choi ${ }^{2}$, and Ho Kyun Lee ${ }^{1}$ \\ Departments of 'Surgery and ${ }^{2}$ Pathology, Chonnam National University Medical School, Gwangju, Korea
}

\begin{abstract}
Adventitial cystic disease (ACD) of the veins is a rare vascular disease. Most cases of venous ACD are located adjacent to the joint area, such as the common femoral, external iliac, and popliteal veins. To the best of our knowledge, 67 cases of venous ACD have been reported, and ACD of the superficial femoral vein (SFV) has never been reported. Herein, we report the case of a 57-year-old male who presented with swelling and discomfort in the left leg. Computed tomography venography revealed multiple cystic lesions in the left distal SFV. The patient underwent cyst excision, which relieved the compression in the vein, although mild stenosis prevailed in the SFV. After a week, thrombosis developed in the popliteal vein. The thrombosis resolved after three months of anticoagulant therapy, and the patient showed no recurrence of ACD during three years of follow-up.
\end{abstract}

Key Words: Adventitial cystic disease, Superficial femoral vein, Computed tomography, Deep vein thrombosis
Received May 4, 2021

Revised June 11, 2021

Accepted June 17, 2021

Published on June 25, 2021

Corresponding author: Ho Kyun Lee

Department of Surgery, Chonnam

National University Medical School, 160

Baekseo-ro, Dong-gu, Gwangju 61469,

Korea

Tel: 82-62-220-6467

Fax: 82-62-227-1635

E-mail:mhaha@hanmail.net

https://orcid.org/0000-0003-3186-7259

Copyright (c) 2021 The Korean Society for Vascular Surgery

This is an Open Access article distributed under the terms of the Creative Commons Attribution Non-Commercial License (http://creativecommons.org/licenses/by-nc/4.0) which permits unrestricted non-commercial use, distribution, and reproduction in any medium, provided the original work is properly cited.

Vasc Specialist Int • https://doi.org/10.5758/vsi.210034

\section{INTRODUCTION}

Adventitial cystic disease (ACD) is a rare vascular condition. Arteries are more commonly affected by ACD than veins. Clinical symptoms of venous ACD are similar to those of deep vein thrombosis (DVT), such as swelling of the affected limb, redness, cramping, and sometimes, ACD causes DVT by compression [1]. Venous ACD develops mostly in the femoral vein, followed by the external iliac and popliteal veins [2]. However, to the best of our knowledge, ACD of the superficial femoral vein (SFV) has not been reported. Herein, we report a case of ACD of the distal SFV. The case report was approved by the Institutional Review Board of Chonnam National University Hospital (approval number CNUH 2021-138), and the need for informed consent was waived due to the retrospective design.

\section{CASE}

A 57-year-old male presented with a 2-year history of swelling in the left leg and heaviness after walking. The patient had no history of comorbidities, trauma, or surgery, except alcoholic liver cirrhosis (Child class B). Physical examination revealed swelling of the left leg without tenderness. Lower extremity computed tomography venography (CTV) had been performed at another hospital for DVT and possible extrinsic compression and revealed multiple cystic lesions compressing the distal portion of the left SFV without DVT (Fig. 1). The proximal cyst had entwined the SFV from the posterior aspect to the medial and anterior aspects of the vein, and the distal cyst was positioned at the posterior aspect of the vein extending down to the level of the adductor hiatus. The cysts were not interconnected. An operation was performed considering the possibility of venous ACD. The SFV was exposed by a medial approach. Two cystic masses were detected along the distal SFV, 

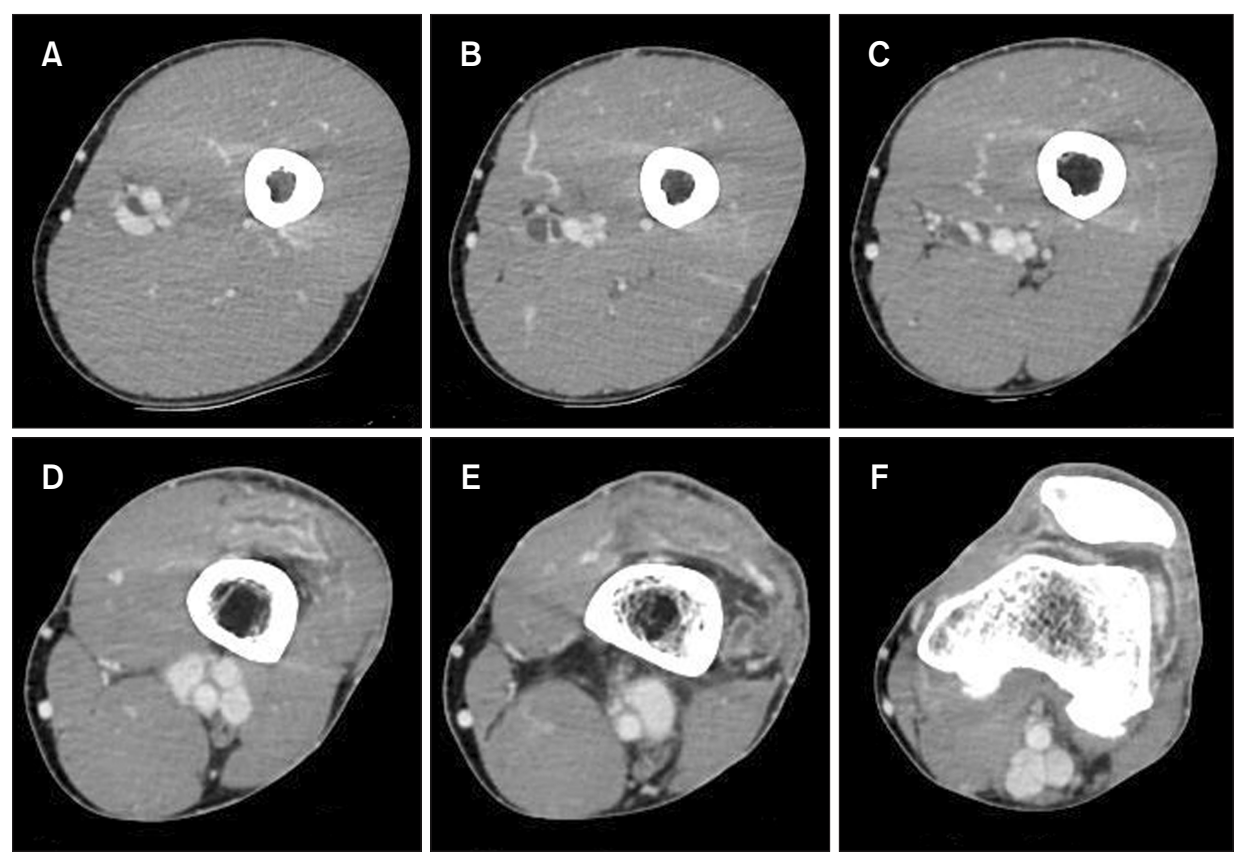

Fig. 1. Computed tomography venography revealed cystic masses of the left distal superficial femoral vein: (A) mid-thigh level, (B) adductor canal level, (C) adductor hiatus level, (D) P1 level, (E) P1 level, (F) P2 level.
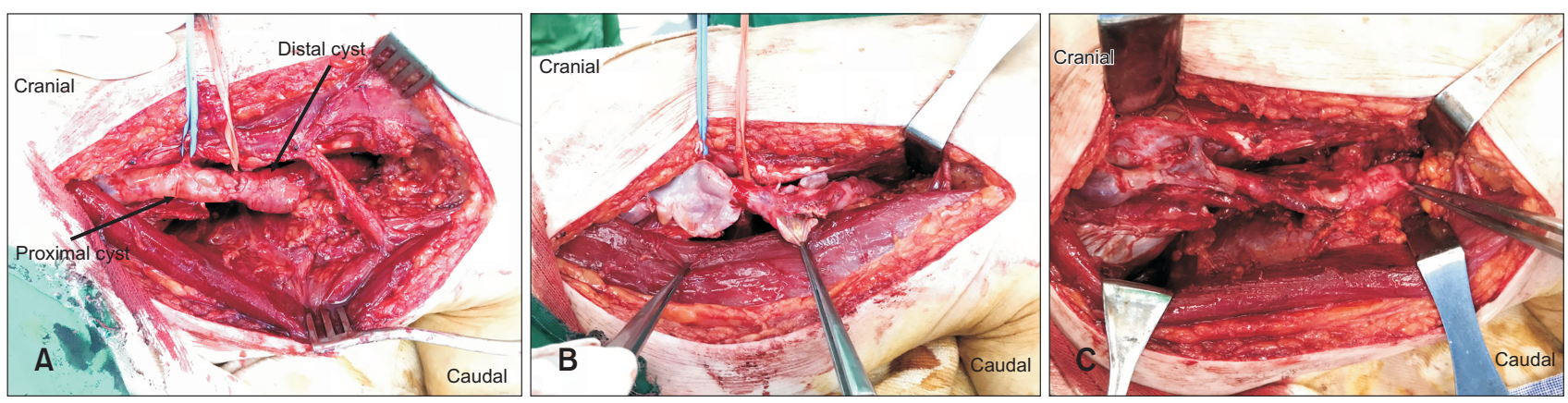

Fig. 2. Operation finding. (A) The mucinous fluid-contained cysts were located at distal superficial femoral vein (SFV). Proximal cyst was spirally encircling the vein and distal cyst was at the posterior aspect of the vein. (B) After opening the cysts, no communications were found with the vessel lumen. (C) After cyst excision, the SFV shows somewhat relieving of the obstructive effect but still remained moderate stenosis.

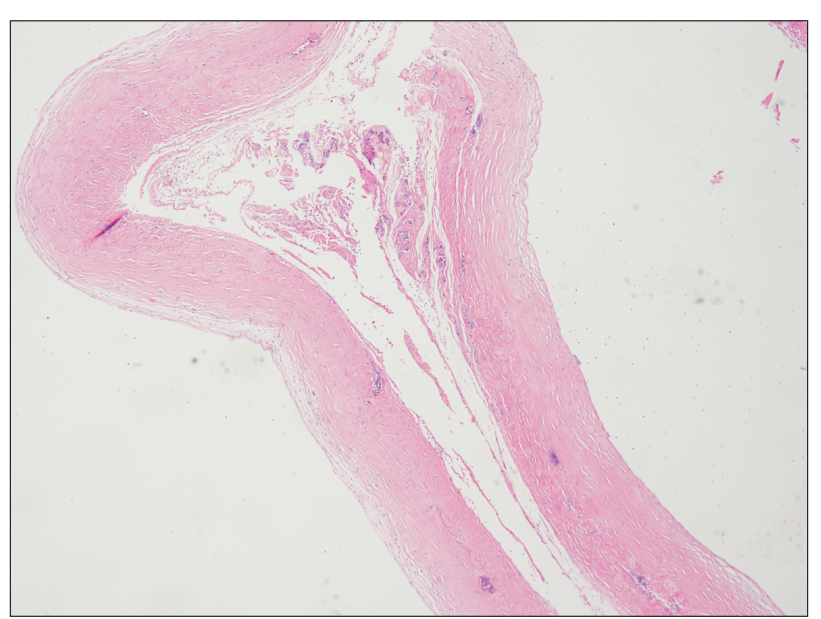

Fig. 3. Histologic finding. Cystic structure consisted of collagen fibers without synovial cells (H\&E stain, $\times 40)$. and no joint connections were identified between the two cystic masses after a thorough investigation. The larger proximal cyst measured $1 \mathrm{~cm} \times 3 \mathrm{~cm}$ and the smaller cyst measured $1 \mathrm{~cm} \times 1.5 \mathrm{~cm}$ (Fig. 2A). The cyst contained mucinous fluid without communication with the vessel lumen. The cyst was excised by resecting its roof portion first and then peeling off the remnant cyst in contact with the vein wall by curettage. During cyst excision, no transmural involvement was observed. After cyst excision, the prevailing stenosis of the SFV was observed, although the obstructive effect was relieved (Fig. 2B, C). Postoperative histology revealed a cystic structure consisting of collagen fibers without any synovial cells; therefore, synovial cyst was excluded from the diagnosis (Fig. 3). Specific staining for synovial markers was not performed because of the absence of synovial cells. Despite the use of low molecular weight hepa- 
rin (LMWH) (enoxaparin, $40 \mathrm{mg}$ twice daily), leg swelling recurred 1 week after surgery. CTV revealed popliteal vein thrombosis beginning at the center of the knee joint space and extending up to the adductor hiatus (Fig. 4). After 3 months of oral anticoagulant therapy with rivaroxaban (15 $\mathrm{mg}$ twice daily for 3 weeks and then $20 \mathrm{mg}$ daily), a followup CT scan revealed resolution of DVT with mild stenosis of the SFV and no recurrence of ACD (Fig. 5). The anticoagulant therapy was discontinued. Follow-up was performed every 6 months, and CTV was performed annually. During 3 years of follow-up, the patient did not present any symptoms such as leg swelling, redness, or cramping and had no recurrence of ACD and new DVT on follow-up imaging.
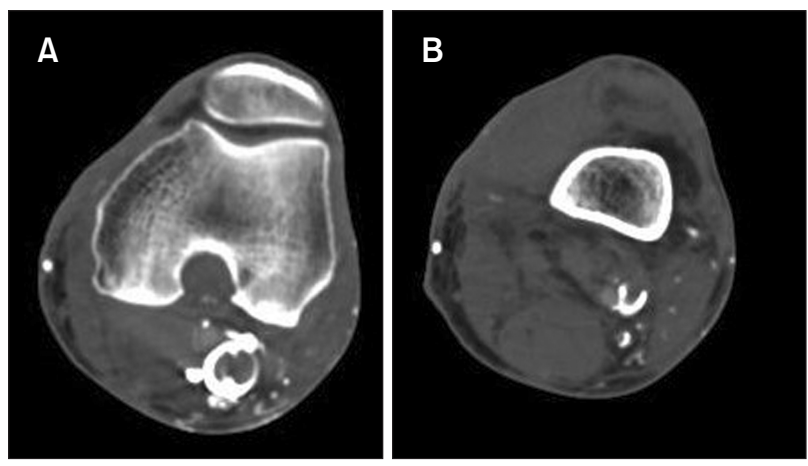

Fig. 4. Computed tomography venography revealed popliteal vein thrombosis at the level of center of knee joint space (A) and extending up to adductor hiatus (B).

\section{DISCUSSION}

ACD is a rare disease that usually affects the arterial system, and venous ACD accounts for 7.4\% of all ACD cases according to a review of 530 studies [3]. Bascone et al. [4] published a review article on venous ACD and reported 45 cases from 1963 to 2016, which amounts to less than one case per year.

Various theories on the etiology and pathogenesis of ACD have been proposed, such as the repeated trauma, de novo adventitial degeneration, developmental and articular theories. However, it is unclear whether the same theories can be applied to arterial and venous ACD [5]. In this case, the patient's age was 57 years, which was greater than the mean age of 46 years reported by Desy and Spinner [3]. This patient had no remarkable medical history except alcoholic cirrhosis and was unemployed and physically inactive. Furthermore, the lesion was located at the distal SFV, which was relatively far from the joint, compared to previously reported cases. Among previously suggested theories, the repeated trauma theory seems applicable to this specific patient. The distal SFV is positioned inside the adductor canal, and the outlet of the adductor canal is prone to compression, which could be the source of repeated trauma [6].

Duplex ultrasonography, CT, magnetic resonance imaging (MRI), and angiography are used in the diagnosis of ACD. Although clinical symptoms may be similar, ACD treatment plans are different from those for DVT or varicose veins; therefore, accurate differential diagnosis based
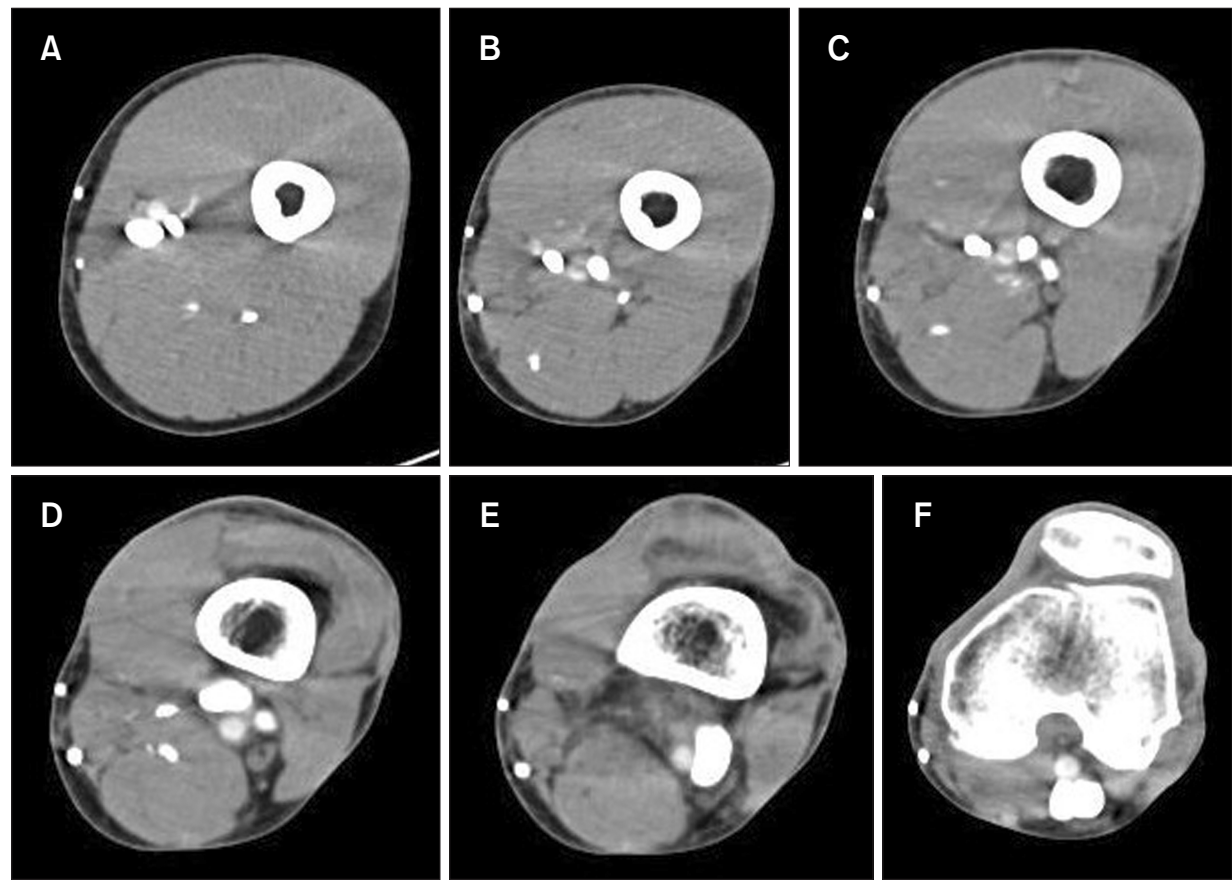

Fig. 5. Follow-up computed tomography venography showed mild stenosis of the left distal superficial femoral vein without recurrence of adventitial cystic disease or deep vein thrombosis: (A) mid-thigh level, (B) adductor canal level, (C) adductor hiatus level, (D) P1 level, (E) P1 level, (F) P2 level. 
on thorough examination is required. Because anatomical factors such as location or adjacent structures are important in selecting the treatment method, imaging modalities such as CT or MRI should be considered.

Various treatment methods for ACD are being investigated, with cyst excision being the most commonly performed one [5]. In case of a high recurrence risk or requirement of complete cyst excision, patch angioplasty or bypass surgeries may be performed. Lim et al. reported that the recurrence rate varies depending on the surgical strategy, and vessel excision with an interposition graft can reduce recurrence compared with simple cyst excision [7]. Cyst excision was performed in our case, and no recurrence was observed during the 3-year follow-up. During the surgery, complete cyst excision of venous ACD was challenging, and we had to peel off the suspected cyst wall with a knife as if almost curetting the cyst wall in contact with the vein. Although tearing of the vessel after cyst excision was not evident, LMWH was administered to prevent thrombosis owing to the possibility of inconspicuous vessel wall injury. However, hematoma occurred at the surgical site, causing a mass effect, which resulted in venous flow disturbance and focal thrombus in the popliteal vein. After 3 months of postoperative anticoagulant therapy, the thrombosis resolved. In our experience, postoperative prophylactic anticoagulation in venous ACD surgery is not necessary unless there is preoperative DVT. Chen et al. [8] had reported the lack of absolute evidence on anticoagulant therapy following an intervention; however, among the reviewed cases, nine patients who had received postoperative anticoagulant treatment showed no recurrence.
ACD occurring in vessels far from the hip or knee joint is rare. In previous reports of ACD at the great saphenous vein, small saphenous vein, and tibial vein, it was located at the ankle joint [9-11], supporting the articular theory of pathogenesis. The main cause of recurrence after surgery of ACD, especially after cyst excision, is a missed connection between the joints [5,7]. Although no joint connection was found during the operation, and no recurrence was observed in the last 3 years, long-term imaging follow-up is required to detect possible recurrence.

\section{CONFLICTS OF INTEREST}

The authors have nothing to disclose.

\section{ORCID}

\author{
Young Sup Yoo \\ https://orcid.org/0000-0002-7773-3005 \\ Yoo Duk Choi \\ https://orcid.org/0000-0002-4385-1759 \\ Ho Kyun Lee \\ https://orcid.org/0000-0003-3186-7259
}

\section{AUTHOR CONTRIBUTIONS}

Concept and design: YSY, HKL. Analysis and interpretation: YSY, HKL. Data collection: YDC. Writing the article: YSY, HKL. Critical revision of the article: YSY, HKL. Final approval of the article: all authors. Statistical analysis: none. Obtained funding: none. Overall responsibility: HKL.
1) Gasparis AP, Wall P, Ricotta JJ. Adventitial cystic disease of the external iliac vein presenting with deep venous thrombosis. A case report. Vasc Endovascular Surg 2004;38:273-276.

2) Levien LJ, Benn CA. Adventitial cystic disease: a unifying hypothesis. J Vasc Surg 1998;28:193-205.

3) Desy NM, Spinner RJ. The etiology and management of cystic adventitial disease. J Vasc Surg 2014;60:235-245, 245.e1-e11.

4) Bascone C, lqbal M, Narh-Martey P, Szuchmacher M, Cicchillo M, Krishnasastry KV. Venous adventitial cystic disease: a review of 45 cases treated since 1963. Int J Vasc Med 2016;2016:5287697.

5) Min SK, Han A, Min S, Park YJ. Inconsistent use of terminology and different treatment outcomes of venous adventitial cystic disease: a proposal for reporting standards. Vasc Specialist lnt 2020;36:57-65.

6) Uhl JF, Gillot C. Anatomy of the Hunter's canal and its role in the venous outlet syndrome of the lower limb. Phlebology 2015;30:604-611.

7) Lim JH, Chung BH, Kang JH, Heo SH, Kim Dl, Kim YW, et al. Surgical strategy to reduce the recurrence of adventitial cystic disease after treatment. Vasc Specialist Int 2019;35:217224.

8) Chen Y, Sun R, Shao J, Li Y, Liu C. A contemporary review of venous adventitial cystic disease and three case reports. Phlebology 2015;30:11-16.

9) Lie JT, Jensen PL, Smith RE. Adventitial cystic disease of the lesser saphenous vein. Arch Pathol Lab Med 1991;115:946-948.

10) Yoshii S, Ikeda K, Murakami H. Cystic, myxomatous adventitial degeneration of a saphenous vein. J Vasc Surg 
1998;27:780-782.

11) Leafblad ND, Wilson TJ, Amrami KK,
Turner NS, Spinner RJ. Cystic adventitial disease of the tibial vein arising from the subtalar joint: a case report. J Foot Ankle Surg 2019;58:377-380. 